operation and no unpleasant effects follow."

This report thus appeared over a year before Morton's demonstration on the use of ether, and I think it is only right to point out that nitrous oxide was in use during the period between Wells's unsuccessful demonstration and Morton's successful one.-I am, etc.,

London W.1

VICTOR GOLDMAN

\section{Ketamine for Burns Surgery}

SIR,-The leading article "Analgesia for Burnt Patients: A Symposium" (20 May, p. 418), gives much needed publicity to a hitherto neglected facet of anaesthetic practice.

The sentence on the paper and discussion on the use of ketamine as an anaesthetic agent for burns surgery is somewhat biased. It is strange to mention one of the less common and least dangerous side effects of ketamine, without making any reference to the drug's unique advantages, especially in the field of burns surgery.-We are, etc.,

S. M. LAIRD M. SAGE

Plastic and Reconstructive Surgery Centre, St. Lawrence Hospital,
Chepstow, Mon

\section{Discharge from Psychiatric Hospitals}

SIR,-In years past the present hospitals for the mentally ill and mentally handicapped served a social, welfare, and custodial, in addition to a medical, function. Scrutiny of the records of older patients in these hospitals reveals that many were admitted at the instigation of their local authorities because they had nowhere else to go or had committed an offence, relatively minor by standards today, or in the case of women, had given birth to illegitimate children. Such people would not be admitted to psychiatric hospitals today.

Unfortunately these patients have remained in the hospitals frequently abandoned or outlived by their relatives and overlooked by the local authorities in whose areas they resided. They continue to occupy expensive hospital beds and obstruct the admission of more urgent cases with a greater and more justifiable need for hospital care. Requests to local authorities for alternative placement will receive sympathetic acknowledgement, but most authorities still have a lack of sufficient alternative lodgings, hostels, or group homes.

It is sometimes contended that it is unkind to move many of the older patients who appear happy and settled in hospitals. The reverse argument that a person should not be admitted to hospital because he has lived most of his life in the general community is rarely cited.

This situation should prompt psychiatric hospitals to compile waiting lists of the patients in their wards who do not need medical and nursing care and who could be discharged to homes or hostels. These waiting lists should be kept up to date and reoularly circulated to social service departments, the regional hospital boards, and the Department of Health and Social Security. This would also serve to emphasize the point that it is not doctors and nurses who are keeping people in psychiatric hospitals, but community outside which is failing to accept them.-I am, etc.,

Meanwood Park Hospital, Leeds

D. A. SPEncer

\section{Intestinal Parasites and Epidemiology of Australia Antigen in Africa}

SIR,-Several recent studies have shown that the incidence of Australia antigen carriers is definitely higher in Africa than in Europe and the U.S.A. While studying the patient records of 94 antigen carriers (black adult Africans) who had been detected in the course of a systematic study of 1,069 randomly chosen subjects at Hôpital Principal of Dakar, we noticed a high incidence of strongyloidiasis and ancylostomiasis in carriers of the antigen. In fact, out of 34 parasitological studies carried out during the hospitalization of these patients we identified eight carriers of Strongyloides and two carriers of Ancylostoma, whereas the average incidence of these parasites, as shown by control study of nearly 10,000 stool specimens over a period of two years, is $2.5 \%$ for strongyloidiasis and approximately $1 \%$ for ancylostomiasis. In spite of the small number of stool analyses done on carriers of the antigen the difference appears to be very significant $(P<0.001)$.

Concomitantly, we discovered that out of 75 of those subjects who had blood counts done there were 13 cases of eosinophiliaabove $10 \%$. In a sampling of adult subjects tested during the period of the study an eosinophil.a higher than $10 \%$ was found in 56 cases out of 600 . Once again the difference is statistically significant $(P=0.05)$.

One can thus find in adult African Australia antigen carriers a statistically significant incidence of intestinal helminthiasis characterized by active cutaneous penetration of larva, and this is corroborated by an equally significant incidence of marked eosinophilia.

Several authors ${ }^{12}$ have suggested that the $\mathrm{Au}$ antigen is excreted in faeces and urine. Because of its great stability and of its resistance to chemical agents this antigen is able to survive in the external environment under the same ecological conditions as those associated with the larvae of Strongyloides, Ancylostoma, and Schistosoma. Inoculation of the antigen could take place at the site of the excoriation produced in the course of active cutaneous penetration of the surface of the skin by these larvae.

This hypothesis would explain the frequent co-existence of the $\mathrm{Au}$ antigen with an intestinal helminthiasis characterized by active cutaneous penetration of the larvae, as noted during the course of our study. It contributes toward an understanding of the particularly high incidence of the $\mathrm{Au}$ antigen in tropical areas, along with other reasons which have been set forth: tattooing (which is not common at the present time in Senegal), ritual practices (such as circumcision), and transmission by arthropod vectors.-We are, etc.

M. BARBotin

Hôpital Principal, Dakar

Senegal

1 Runcan, V., State, Z., and Dumitrescu, M., 2 Tripatzis, I., and Horst, H. G., Nature, 1971,
Oral Prostaglandins and Amniotomy

SIR,-Mr. W. Barr and Dr. W. C. M. K. Naismith (22 April, p. 188) describe the first results of using oral prostaglandins to induce labour in centres in the United Kingdom. The results from this department (22 April p. 191) were an evaluation of amniotomy and oral prostaglandin $\mathbf{E}_{2}$ titration in both primigravid and multigravid patients, while that of Mr. Barr and Dr. Naismith mainly assessed the use of prostaglandin $\mathbf{E}_{2}$ and $\mathrm{F}_{2} \alpha$ in multigravida. In the latter amniotomy was not undertaken until the "active phase" of labour was established.

I would like to stress the value of undertaking amniotomy at the onset of induction in accelerating the progress of labour when used in conjunction with these agents. From consideration of the results of these studies it is apparent that even in multigravida the time to delivery was considerably shorter when early rupture of the membranes was performed. For those with a singleton pregnancy the mean induction delivery interval was 7 hours 26 minutes for the 16 multigravid patients in the former study, compared with 11 hours 9 minutes for the 22 out of 24 patients in whom induction of labour was successful in the latter.

The advantage of using the combined regimen is seen in the Table where the induction delivery interval for multigravid patients having amniotomy and oral prostaglandin $\mathrm{E}_{2}$ is compared with that of an unselected group in whom labour was induced by amniotomy alone. The difference between these times is significant $(t=2.24, P<0.05)$.

Induction of Labour in Multigravid Patients

\begin{tabular}{|c|c|}
\hline & $\begin{array}{l}\text { Induction-deliverv Interval } \\
\text { Mean } \pm \text { S.E.M. }\end{array}$ \\
\hline $\begin{array}{l}\text { Amniotomy and Oral } \\
\text { Prostaglandin } \mathrm{E}_{\text {, }} \\
(\mathrm{n}=16)\end{array}$ & $7 \mathrm{hr} .26 \mathrm{~min} \pm 1.24$ \\
\hline$\underset{(n=23)}{\text { Amnio*omy Alone }}$ & $12 \mathrm{hr} .31 \mathrm{~min} \pm 1.34$ \\
\hline
\end{tabular}

While it is appreciated that the protocol followed by Mr. Barr and Dr. Naismith was deliberately planned so that the effectiveness of using oral prostaglandins as a uterine stimulant for the induction of labour could be evaluated in detail in the absence of amniotomy, such a practice would appear to have limitations for routine clinical use for the time to delivery reported above from this study is not muah shorter than that described here for amniotomy alone.-I am, etc.,

IAN CRAFT

Department of Obstetrics and Gynaecology.

King ton Hospital.

Kingston upon Thames,

Surrey

\section{Rheumatoid Arthritis and Malabsorption}

SIR,-In vour leading article on "Rheumatoid Arthritis and Malabsorption" (22 April, p. 181) you note that the drug treatment of malabsorption in this situation requires further investigation.

With G. Andrews I described 1 the use of D-penicillamine $1.8 \mathrm{~g}$ per day in conjunction with pyridoxine $50 \mathrm{mg}$ per day in a desperately ill patient with rheumatoid arthritis, malabsorption, and amyloidosis, who had responded to no other therapy 